\title{
A dimensão prescrita e real de práticas de profissionais de saúde no contexto da segurança do paciente
}

\author{
Dimension prescribed and realyity of practices of health professionals in the context of patient safety \\ La dimensión prescrita y real de prácticas de profesionales de la salud en el contexto de la \\ seguridad del paciente
}

Andreia Guerra Siman'; Maria José Menezes Brito"

\begin{abstract}
RESUMO
Objetivo: compreender a dimensão prescrita e real das práticas de profissionais de saúde no contexto da segurança do paciente. Método: estudo de caso qualitativo. Os participantes foram 31 profissionais: membros do Núcleo de Segurança do Paciente e a equipe de enfermagem de um hospital de ensino, localizado em Minas Gerais, Brasil. Os dados foram coletados por meio de entrevistas com roteiro semiestruturado, observação e dados secundários. Foi realizada triangulação de dados e a análise de conteúdo. O projeto foi aprovado no Comitê de Ética em Pesquisa, CAAE 44109015.0.0000.5149. Resultados: as transformações ocorridas, como a criação do núcleo, plano de segurança do paciente e protocolos, foram evidenciadas mais na concepção prescritiva. A segurança do paciente não foi priorizada como diretriz estratégica do hospital. Falta capacitação da equipe e avaliação das ações. Conclusão: a segurança do paciente foi evidenciada mais no âmbito prescrito. $O$ trabalho realizado não corresponde exatamente àquele proposto pelas regras e protocolos.
\end{abstract}

Descritores: Segurança do paciente; hospitais; gestão da qualidade; serviços de enfermagem.

\section{ABSTRAT}

Objective: to understand the prescribed and real dimensions of daily patient safety practice by health personnel. Method: the participants in this qualitative case study were 31 members of the Patient Safety Center and the nursing staff at a teaching hospital in Minas Gerais, Brazil. Data collected in 2015 by scripted, semi-structured interview, were complemented by observation and secondary data. Data triangulation and content analysis were used. The project was approved by the research ethics committee (CAAE 44109015.0.0000.5149). Results: changes were more evident in the prescriptive conception (introduction of the center, patient safety plan and protocols). Patient safety was not prioritized as a strategic guideline at the hospital, where team training and evaluation of actions were lacking. Conclusion: patient safety was more evident as a prescription. The work being done does not correspond exactly to what is proposed by the rules and protocols.

Descriptors: Patient safety; hospitals; quality management; nursing services.

\section{RESUMEN}

Objetivo: comprender la dimensión prescrita y real de las prácticas de profesionales de salud en el contexto de la seguridad del paciente. Método: estudio de caso cualitativo. Los participantes fueron 31 profesionales: miembros del Núcleo de Seguridad del Paciente y el equipo de enfermería de un hospital de enseñanza, ubicado en Minas Gerais, Brasil. Los datos fueron recolectados por medio de entrevistas, con guion de entrevistas semiestructuradas, observación y datos secundarios. Se realizó la triangulación de datos y el análisis de contenido. El proyecto fue aprobado en el Comité de Ética en Investigación, CAAE 44109015.0.00.00.500. Resultados: las transformaciones que ocurrieron, como la creación del núcleo, plan de seguridad del paciente y protocolos, se volvieron más evidentes en la concepción prescriptiva. La seguridad del paciente no fue priorizada como directriz estratégica del hospital. Falta capacitación del equipo y evaluación de las acciones. Conclusión: la seguridad del paciente quedó más evidente en el ámbito prescrito. El trabajo realizado no corresponde exactamente a aquél propuesto por las reglas y los protocolos.

Descriptores: Seguridad del paciente; hospitales; gestión de la calidad; servicios de enfermería.

\section{INTRODUÇÃO}

O objeto deste estudo"l' são as práticas cotidianas de profissionais de saúde no contexto da segurança do paciente, as dimensões prescritas e reais em um hospital de ensino de Minas Gerais.

No Brasil, considerando a magnitude dos eventos adversos, em 2013 foi instituído o Programa Nacional de
Segurança do Paciente ${ }^{1}$ e publicada uma resolução que institui ações para a promoção da segurança do paciente e a melhoria da qualidade nos serviços de saúde ${ }^{2}$. Cada serviço deve constituir o Núcleo de Segurança do Paciente (NSP) e disponibilizar recursos humanos, financeiros, equipamentos, insumos e materiais. 
Nesse sentido, os profissionais de saúde e os gerentes, necessitam da implantação e apropriação de ferramentas para garantir que todas as etapas de um processo sejam cumpridas e, consequentemente, falhas sejam evitadas. Entretanto, como tem sido a implantação do núcleo? É uma estratégia suficiente para subsidiar mudanças nas práticas dos profissionais? A legislação, o controle, a organização, as normas estratégicas, os protocolos garantem mudança nas práticas cotidianas dos profissionais?

As padronizações podem não garantir adequadamente a segurança do paciente, e há evidências de que são necessários mais esforços para melhorar os processos dentro das instituições de saúde, no foco da segurança ${ }^{3}$. Parte-se do pressuposto que há lacunas no que tange à instituição de protocolos, à execução efetiva e à avaliação do processo para subsidiar ações gerenciais e assistências, como um distanciamento entre as dimensões prescritas do planejamento das práticas de segurança do paciente no hospital e o real trabalho dos profissionais. Nessa perspectiva, os gerentes e administradores das instituições devem diminuir barreiras e reunir esforços para desenvolver uma cultura de segurança por meio da informação e aprendizagem.

O objetivo deste estudo foi compreender a dimensão prescrita e real das práticas de profissionais de saúde no contexto da implantação da segurança do paciente.

\section{REVISÃo DE LITERATURA}

Iniciativas voltadas para a segurança do paciente começaram com a publicação do relatório To err is human, o qual apontou que até 98 mil mortes por ano nos Estados Unidos poderiam ser evitáveis devido a erros médicos ${ }^{4}$ gerando alerta mundial às instituições sanitárias.

A Classificação Internacional de Segurança do Paciente relaciona segurança do paciente à redução de riscos de danos ou lesões, associado ao cuidado em saúde, dentro de uma aceitação mínima ${ }^{5}$.

Neste estudo, foram adotadas algumas definições: risco, como a probabilidade de ocorrência de um acidente; erro é a falha, ação que ocorre fora do planejado ou aplicação incorreta do plano; os eventos adversos (EAs) são considerados como qualquer dano ou lesão causado ao paciente pela intervenção da equipe de saúde ${ }^{5}$.

Pesquisas têm demonstrado que as práticas cotidianas dos profissionais de saúde têm sido marcadas por elevadas taxas de erro, denúncia de lesões em pacientes, falhas nos sistemas de atendimento hospitalar, danos permanentes e mortes. Nesse sentido, práticas inadequadas, inseguras e negligentes afetam um a cada dez pacientes em média em países desenvolvidos ${ }^{4,6}$.

No presente estudo, optou-se por trabalhar com o conceito de prática no sentido mais amplo, como práticas sociais dentro da perspectiva praxeológica. Trata-se de uma abordagem que possa agrupar a análise de gestão, os níveis institucionais, organizacional e comportamental permitindo as interseções com as ações humanas ${ }^{7}$.

\section{Metodologia}

O estudo é de abordagem qualitativa, delineado pela estratégia de pesquisa estudo de caso, que objetiva analisar uma unidade social, buscando responder como e por que os fenômenos ocorrem. A natureza do problema e os detalhes do fenômeno a serem analisados justificam a escolha da realização de um estudo de caso ${ }^{8}$.

Para definição do cenário de estudo, levou-se em consideração ser um hospital zeloso pela a qualidade e segurança do paciente, participante da Rede de Hospitais Sentinela, e, portanto, possuir uma comissão de gerenciamento de riscos. Trata-se de um hospital filantrópico, vinculado com hospital de ensino, localizado na Zona da Mata de Minas Gerais, Brasil. Possui 116 leitos e média de 5.232 pacientes-dia ao mês. É um serviço de referência de uma microrregião composta por nove municípios e possui seis alas de internação.

Os participantes da pesquisa foram profissionais membros do NSP e a equipe de enfermagem das alas de internação - clínica médica e cirúrgica, tendo por critério de inclusão possuir, no mínimo, um ano de trabalho. A escolha por entrevistar a equipe das alas de internação se deu com base em resultados de uma pesquisa, na qual o objetivo foi avaliar a incidência de eventos adversos em hospitais no Brasil. Seus resultados apontaram que a enfermaria foi o local com maior frequência de eventos adversos $(48,5 \%)^{9}$. A escolha por profissionais do NSP se deu por se entender que são eles quem gerenciam e prescrevem as ações para alcançar a segurança do paciente.

O NSP era composto por 14 membros e todos foram convidados a participar, no entanto, dois profissionais estavam de licença médica, totalizando, 12 sujeitos. Os enfermeiros das alas de internação constituíam um grupo de nove profissionais, plantão noturno e diurno, sendo que dois não corresponderam ao critério de inclusão, reduzindo o conjunto a sete participantes. Quanto aos técnicos de enfermagem, utilizou-se o critério de saturação, o qual interrompe as entrevistas quando os dados coletados se tornam repetitivos e redundantes. Os dados saturaram na $12^{\text {a }}$ entrevista. No total, participaram da pesquisa 31 profissionais.

A coleta de dados foi realizada no período de maio a novembro de 2015, com dados primários e secundários. Os dados secundários foram obtidos por meio de pesquisa documental (relatórios, protocolos, indicadores e registros da qualidade e plano de ação do NSP), possibilitando o resgate de fontes prescritas. Os dados primários foram coletados mediante a realização de entrevistas, com roteiro semiestruturado, e observação com registro em diário de campo. A observação e as entrevistas promoveram o resgate de fontes reais. 
As entrevistas foram gravadas e transcritas na íntegra e tiveram duração de dez a 100 minutos. Foram numeradas de acordo com a sequência em que ocorreram e codificadas com a sigla NSP, E (enfermeiro) e TE (técnico de enfermagem). As perguntas buscavam compreender aspectos como conhecimento sobre segurança do paciente, avaliação da segurança do paciente na instituição, práticas e notificações para alcançar a segurança, dificuldades e facilidades para alcançar as metas internacionais e atividades do núcleo.

Para tratamento dos dados foi utilizada a análise de conteúdo, especificamente a categoria temática, a qual consiste em um conjunto de técnicas de análise de comunicações, realizada em torno de três pólos cronológicos: a pré-análise, a exploração do material e tratamento dos resultados ${ }^{10}$. Segundo procedimentos sistemáticos, foram definidas unidades de codificação ou de registro que, por sua vez, foram agrupadas em duas categorias.

Todos os participantes assinaram o Termo de Consentimento Livre e Esclarecido, em cumprimento da Resolução no 466/12, do Ministério da Saúde, sendo o projeto aprovado pelo Comitê de Ética em Pesquisa do Hospital e pelo Comitê de Ética em Pesquisa da Universidade Federal de Minas Gerais, CAEE no 44109015.0.0000.5149.

\section{RESULTADOS E DISCUSSÃO}

São analisadas a seguir as duas categorias emergentes do estudo.

\section{Histórico da implantação do núcleo de segurança do paciente}

Esta categoria foi construída, principalmente, a partir da análise documental e da observação de campo. Em 2010, o hospital iniciou o Processo de Acreditação Hospitalar, pleiteando o certificado de Acreditado, nível 1 da Organização Nacional de Acreditação (ONA). Foi organizado o setor da qualidade, e, em 2010, solicitada a visita diagnóstica de uma Instituição Acreditadora Credenciada. Na ocasião, foi emitido um relatório sugerindo várias modificações no âmbito de estruturas, processos e resultados. Entretanto, a instituição passou por algumas dificuldades, o que impossibilitou dar prosseguimento ao processo.

A acreditação hospitalar é uma das estratégias utilizada para melhorar a qualidade e minimizar a ocorrência de erros. As instituições precisam cumprir normas que incidem sobre a segurança do paciente, sobre a padronização de procedimentos, mudanças no gerenciamento do trabalho, criação de gestão de riscos, protocolos e indicadores ${ }^{11,12}$.

A segurança é considerada uma das dimensões da qualidade ${ }^{1}$, que são indissociáveis, e os serviços de saúde que diminuem a um mínimo possível os riscos de dano ao paciente (segurança) estão aumentando a qualidade da assistência prestada ${ }^{13}$.
Em 2012, a instituição foi credenciada à Rede Brasileira de Hospitais Sentinela, coordenada pela Agência Nacional de Vigilância Sanitária, que visa à prevenção de riscos associados ao consumo de produtos sujeitos à vigilância sanitária. Os hospitais vinculados devem notificar, monitorar eventos adversos, queixas técnicas de produtos e promover medidas para reduzí-los. A participação é voluntária e a principal finalidade é a notificação de eventos adversos ${ }^{14}$.

O hospital tinha o Programa de Fortalecimento e Melhoria da Qualidade dos Hospitais (Pro-HOSP) do Sistema Único de Saúde (SUS), que já exigia a criação da comissão de gerenciamento de risco. Seus membros compuseram o NSP, criado em maio de 2013, sendo acrescidos outros profissionais. Nesse período, foi realizado o mapeamento de risco de cada setor e instituídas as fichas de notificações de queixas técnicas.

Ressalta-se que é aceitável que a direção do serviço de saúde empregue a estrutura de comitês, comissões, gerências, coordenações ou núcleos já existentes para o desempenho das atribuições do núcleo².

O NSP foi nomeado pelo diretor administrativo com nove enfermeiros representantes de todos os setores; um técnico de enfermagem; um nutricionista; o coordenador da recepção; coordenador da manutenção; o farmacêutico e gerente de apoio operacional. Chama a atenção o fato de não haver um representante médico, o que seria fundamental para atender o princípio de disseminação sistemática da cultura de segurança. Também não foram identificadas comissões afins atuando como membros consultivos do núcleo ${ }^{15}$.

Apesar da criação do núcleo, a instituição manteve suas notificações, ações preventivas, educativas com foco nas queixas técnicas, e só em 2015 instituiu a ficha de notificação de EA internos. O gerente de riscos atualiza o registro das notificações no Sistema nacional de Notificação da Vigilância Sanitária (NOTIVISA). Estudo aponta que $50 \%$ das notificações de EAS e queixas técnicas são provenientes da Rede Sentinela ${ }^{14}$.

Conforme preconiza a RDC no 36, a instituição em estudo elaborou um Plano de Segurança do Paciente (PSP), documento que aponta situações de risco e descreve as estratégias e ações para alcançar as metas internacionais de segurança, visando à prevenção e à mitigação dos incidentes ${ }^{2}$.

Estudo indica que o cumprimento de identificação do paciente, a aplicação do check list para cirurgia segura, da segurança na administração de medicamentos, entre outros, representam melhoria dos processos nas instituições de saúde, visto que, para o atendimento dessas exigências, é necessária a adequação de diversos processos gerenciais e assistenciais, permitindo não só a segurança dos pacientes como também dos profissionais ${ }^{16}$.

A respeito de protocolos, constavam descritos alguns, como o de prevenção de queda e de úlcera por pres- 
são; cirurgia segura; identificação do paciente; higienização das mãos; terapia enteral e parenteral; administração de hemocomponentes. Não havia protocolo de administração de medicação com a justificativa de não haver uma farmacêutica clínica. Esses achados são consistentes com estudo realizado com 14 gerentes de risco, o qual apontou que as iniciativas menos utilizadas para a segurança do paciente são as relacionadas ao controle e prevenção de EAs com medicamentos. O farmacêutico clínico ainda não é uma realidade nos hospitais brasileiros ${ }^{17}$.

Em relação à observação no setor de internação, as seis unidades são localizadas do segundo ao quinto andar. Foram visualizados cartilhas e cartazes do Ministério da Saúde sobre segurança do paciente, e pasta contendo o Procedimento Operacional Padrão (POP) de notificação de EAs.

Foi possível observar técnicos de enfermagem atarefados e mais sobrecarregados que outros profissionais. Geralmente os profissionais estavam agitados, muitos residentes, locais de grande circulação de pessoas, muitos profissionais manipulando os prontuários. As enfermarias apresentavam uma grande variedade diagnóstica e de condições clínicas dos doentes. Ressalta-se que essa variedade de condições clínicas pode dificultar a padronização das condutas, além disso, a demanda geralmente é maior que a capacidade de atendimento de qualidade ${ }^{18}$.

Em relação à rotina de trabalho, identificou-se a desorganização no trabalho - técnicos tomavam decisões sobre rotinas, escalas e divisões de tarefas, sendo a falta de organização do serviço um aspecto que está relacionado aos $\mathrm{EAs}^{19}$. Os técnicos de enfermagem desenvolviam ações de higiene dos pacientes e administração de medicações. Em algumas situações, os técnicos se reportavam ao médico. O enfermeiro assumia dois setores localizados em andares distintos, dificultando seu trabalho.

Observou-se comunicação escassa entre a equipe multiprofissional. Utilizavam caderno administrativo, passagem de plantão e mural de internação (instrumentos administrativos convencionais), mas parecia não haver planejamento e condução do processo de trabalho. As condutas dos enfermeiros eram pontuais e marcadas pelas tentativas de implantação da Sistematização da Assistência de Enfermagem (SAE). Identificaram-se lacunas no conhecimento e ausência de ações pautadas nos princípios da qualidade e segurança. A enfermagem se sentia insegura devida à falta de recursos humanos, materiais e de ausência de manutenção de equipamentos.

Esses achados remetem à prática do enfermeiro em estabelecer relações pessoais como propriedade indispensável para atingir os resultados, monitorar os EAs, trabalhar pela qualidade e melhoria da segurança e assistência prestada ao paciente. Reforça-se a importância de ele atuar frente aos problemas em busca de soluções que proporcionem melhorias para a prática clínica como revisar processos assistenciais ${ }^{20}$. É preciso incluir o estudo sobre liderança e realizar treinamentos e educação continuada, com todos os profissionais envolvidos no processo de cuidado ao paciente ${ }^{21,22}$.

\section{A segurança do paciente: do imaginário ao real}

O estudo revelou uma distinção entre o prescrito e o real para o alcance da segurança do paciente. A despeito de existir, no âmbito prescrito, um núcleo bem estruturado, inferere-se que a sua concretização ainda não ocorreu, como se ainda estivesse no imaginário dos seus idealizadores, ou apenas como cumprimento de normas para fins fiscalizadores.

O NSP é uma instância da instituição que tem como objetivo disseminar a cultura de segurança e a análise de incidentes notificados, identificando suas causas e adotando estratégias de prevenção ${ }^{2}$. Entretanto, alguns profissionais desconheciam a existência do núcleo e suas práticas, o que evidencia que formalmente ele estava implantado, mas, com pouco impacto no âmbito real:

Não conheço. Nem sabia desse núcleo, para ser sincero. (TEC 21)

Não conheço na instituição. Ninguém nunca se apresentou para mim. Só se apresentou para alguma outra enfermeira. (E3)

Eu desconheço alguma ação voltada especificamente para a segurança do paciente. Não é formalmente realizada. (E1)

Destaca-se a ausência de relação direta entre os membros do núcleo e os demais profissionais de saúde, não sendo reconhecidos como líderes:

Essa semana a gente teve um problema, marquei a reunião com as duas e conseguimos resolver. Foi uma notificação que eu recebi e as meninas estranharam. O que você está fazendo aqui? O problema é entre os dois setores e eu como enfermeira do controle de infecções não tinha nada a ver, mas eu como gerente de risco tinha (NSP2).

Durante a observação de campo e a análise documental, foi possível identificar que as normas e os registros do núcleo constavam no âmbito prescritivo, mas com ações tímidas e influenciaram em reduzidas mudanças na prática profissional. É fundamental contar com recursos adicionais para melhorar a segurança do paciente nos hospitais públicos e prioriza-la como diretriz estratégica, que é definida pela liderança e administração do hospital ${ }^{23}$. Entretanto, os dados corroboram a afirmativa - o cenário brasileiro dos hospitais ainda está baseado em redução de custos $^{13}$. Recomenda-se promover a segurança do paciente e desenvolvê-la em todos os níveis da organização, com líderes dando direção às ações de melhoria ${ }^{21}$.

Os participantes revelaram insatisfação por não haver feedback da alta administração, após a análise dos EAs. As organizações de saúde devem medir sua cultura de segurança, dar retorno à liderança e à equipe, o que poderá reduzir riscos, buscando melhorias ${ }^{21}$.

Portanto, o núcleo deve ser operacional e ter apoio da administração. Houve várias notificações de quedas. Só 
preencho o papel. Já teve outras coisas mais graves. Depois que notifico aqui, não tive nenhum feedback. (E14)

Outro aspecto que evidencia o distanciamento entre o prescrito e o real foi o fato de os membros do NSP terem sido capacitados, mas não multiplicaram esse conhecimento para os demais profissionais, confirmando uma desarticulação do núcleo com demais profissionais, acarretando descontinuidade dos processos:

O curso que nós fizemos, tinha que expor para todos os profissionais. Foi em São Paulo, foram oito pessoas que fundaram o núcleo. Mas o que fizemos? Acho que tínhamos que chegar e passar [esses conhecimentos] para todos os funcionários. (NSP5)

O que mais temos tentado fazer é notificar. Acredito que tem muita gente, a maioria das técnicas, que não sabem. Nós sabemos por que somos o núcleo. (NSP12)

A comunicação eficaz é determinante para a segurança do paciente, sendo fundamentais o trabalho e a diversidade interdisciplinar, compartilhando saberes, com treinamento e educação adequada para desempenho das funções ${ }^{21}$.

Em relação à notificação de EAs, era realizada manualmente e em impresso próprio, no entanto, $58 \%$ dos participantes da pesquisa desconheciam o processo e não tinham acesso à ficha de notificação. Principalmente, os técnicos de enfermagem relataram desconhecer a existência do impresso. As auditorias dos EAs eram realizadas quase sempre no dia da ocorrência, no entanto, suas implicações geralmente eram punitivas sem alterar a prática:

Nunca aconteceu comigo queda de paciente. Nenhum outro evento. Tem um para punção venosa lá na sala, um impresso próprio. Agora, nunca aconteceu comigo. Nunca vi outro impresso. (E20)

Que eu saiba não. Se tiver alguma folha aí para notificar, eu não sei. (TE21)

Conhecer os riscos institucionais é necessário para evita-los e gerencia-los ${ }^{24}$. No entanto, faz-se necessária uma abordagem do erro de forma sistêmica ${ }^{25}$ e uma avaliação baseada em indicadores ${ }^{25}$

A respeito do plano, observou-se que não continha ações e metas para alcançar a segurança do paciente. Não foram evidenciados uso de ferramentas de avaliação e nem estratégias para diminuir os EAs:

Eu vou ser muito sincera, se teve plano de ação eu não me lembro de como ele foi executado. Tem alguns protocolos que eu ajudei a fazer, mas já tem muito tempo, foi de prevenção de ulcera e não me lembro. (NSP 9)

Eu já vi um pouco sobre o regimento interno, mas, o plano de ação, eu não cheguei a ver. (NSP10)

Os dados apontam que não houve uma construção coletiva do plano e nem um trabalho multidisciplinar, perdendo-se a oportunidade de valorizar o conhecimento de cada profissional, dificultando a disseminação e adesão às propostas de aprimoramento dos serviços assistenciais. O plano deve expor atividades de monitoramento, objetivando detectar problemas e controlar a manutenção das melhorias atingidas ${ }^{13}$.

Outro destaque, e um dos primeiros resultados do trabalho do NSP, é representado pelos protocolos de segurança. Embora a análise documental tenha evidenciado a existência desses protocolos, as práticas dos profissionais dizem respeito a ações isoladas e pessoais. Os profissionais relataram:

Eu tento fazer isso informalmente. Como não existe formalmente, eu tento conferir tudo, conferir se a medicação está correta, conferir se os materiais estão corretos, orientar os funcionários a fazer a conferência correta. (E1)

Não temos o protocolo ainda, mas eu acho que os meninos conseguem identificar mais a questão da farmacovigilância que o evento interno. (NSP2)

A análise dos dados permite inferir que os protocolos estavam no plano prescritivo da instituição, mas na prática eram desconhecidos. Enfatiza-se que os protocolos e a SAE são processos que auxiliam a padronizar o tratamento, minimizar o erro e promover a segurança do paciente ${ }^{26}$. 0 descumprimento desses protocolos assistenciais é um demonstrativo de falta de qualidade e segurança. Sua implantação melhora os resultados do tratamento e a qualidade dos cuidados, evitando danos mais graves ${ }^{27}$. Os achados sinalizam que há necessidade de maiores investimentos técnico-científicos e promoção da cultura de segurança na alta direção, semelhantemente em estudo realizado recentemente ${ }^{28}$.

Os resultados fornecem contribuições inovadoras para maior compreensão dos desafios nos hospitais públicos - a busca de uma prática cotidiana voltada para a segurança do paciente. Além disso, o trabalho realizado nem sempre corresponde exatamente àquele prescrito, proposto pelas regras e protocolos. É grande e imprevisível a variabilidade da ação humana, no momento da execução da tarefa, pois o trabalho se apresenta revestido de complexidade e envolvido pela atividade humana ${ }^{29}$. O caráter qualitativo desta pesquisa e a ausência de estudos similares limitam a comparação dos resultados.

\section{CONCLUSÃo}

O estudo mostrou que as transformações ocorridas são mais evidenciadas nas concepções prescritivas do que na prática. Faz-se necessária uma reorientação efetiva, por parte do NSP, para a adoção de práticas de segurança do paciente, não só pela equipe de enfermagem, mas por todos os profissionais de saúde. Os resultados indicam um longo caminho a ser percorrido para atingir os objetivos da Organização Mundial de Saúde no alcance das metas de segurança do paciente.

Teoricamente os participantes referiram práticas embasadas em metas internacionais de segurança do paciente, no entanto, a realidade se mostrou para aquém do recomendado; uma dura realidade que pre- 
cisa ser transformada com apoio e envolvimento da alta direção, atuação efetiva do núcleo e uma proposta de educação permanente.

Reduzir riscos envolve um trabalho de todas as áreas do hospital e não só uma atribuição do núcleo. Entretanto, o cenário brasileiro ainda está baseado em redução de custos e não há prioridade em segurança do paciente. São barreiras que precisam ser vencidas para garantir uma cultura de segurança do paciente.

As principais limitações da pesquisa são decorrentes da metodologia adotada, pois estudos de caso não permitem generalizações, assim, a realidade aqui encontrada não representa, necessariamente, a realidade de outras instituições.

\section{REFERÊNCIAS}

1. Ministério da Saúde (Br). Portaria no 529, de 10 de abril de 2013 . Institui o Programa Nacional de Segurança do Paciente (PNSP). Diário Oficial da União, 2 abr 2013 [citado em 23 nov 2017]. Disponível em: http://bvsms.saude.gov.br/bvs/saudelegis/ gm/2013/prt0529_01_04_2013.html

2. Ministério da Saúde $(\overline{B r})$. Resolução da Diretoria Colegiada no36 de 25 de julho de 2013. Institui ações para a segurança do paciente em serviços de saúde e dá outras providências Brasília; 2013 [citado em 23 nov 2017]. Disponível em: http://bvsms.saude. gov.br/bvs/saudelegis/anvisa/2013/rdc0036_25_07_2013.html 3. Antonoff MB, Berdan EA, Kirchner VA, Krosch TC, Holley CT, Maddaus MA, D'Cunha J. Who's covering our loved ones: surprising barriers in the sign-out process. Am. J. Surg[Online]. 2013;205(1):77-84. Doi: https://doi.org/10.1016/j.amjsurg.2012.05.009.

4. Kohn LY, Corrigan JM, Donalson MS. Committee on quality of health care in america. To err is human: building a safer health system. Washington (DC): National Academy; 2000.

5. Runciman W, Hibbert P, Thomson R, Van DST, Sherman H, Lewalle P. Towards an international classification for patient safety: key concepts and terms. Int. J. Qual. Health Care[Online]. 2009;21(1):18-26. Doi: 10.1093/intqhc/mzn057.

6. World Health Organization. World alliance for patient safety. Forward program. 2006-2007. [cited in 2017 Apr 15]. Available from: http://www.who.int/patientsafety/information_centre/ WHO_EIP_HDS 2006.1.pdf.

7. Reed M. Sociologia da gestão. Lisboa (Pt): Celta; 1997.

8. Yin RK. Estudo de caso. Planejamento e métodos. Tradução de Daniel Grassi. 5a ed. Porto Alegre (RS): Bookman; 2015.

9. Mendes W, Martins M, Rozenfeld S, Travassos C. The assessment of adverse evenst in hospitals in Brazil. Int J Qual Health Care[Online]. 2009; 21(4):279-284. Doi: 10.1093/intqhc/mzp022. 10. Bardin L. Análise de conteúdo. Lisboa (Pt): Edições 70; 2011. 11.Sousa P. Patient safety: a necessidade de uma estratégia nacional. Acta Med. Port. 2006;19: 309-18.

12.Siman AG, Cunha SGS, Brito MJM. Mudanças nas ações gerenciais após a acreditação hospitalar. Rev. Rene[Online]. 2016 [citado em 7 jan 2018]; 17(2):165-75. Doi: http://dx.doi. org/10.15253/rev\%20rene.v17i2.2992.

13. Ministério da Saúde (Br). Agência Nacional de Vigilância Sanitária. Assistência segura: uma reflexão teórica aplicada à prática. Série segurança do paciente e qualidade em serviços de saúde.
Brasília (DF): ANVISA; 2013. [citado em 16 maio 2017]. Disponível em: http://www20.anvisa.gov.br/segurancadopaciente/images/ documentos/livros/Livro1-Assistencia_Segura.pdf

14. Ministério da Saúde (Br). Agência Nacional de Vigilância Sanitária. Estratégias para segurança do paciente em hospitais e clínicas. Brasília (DF): ANVISA; 2010 [citado em 15 nov 2017]. Disponível em: www.anvisa.gov.br

15. Nascimento Serra J, Barbieri AR, Cheade MFM. Situação dos hospitais de referência para implantação funcionamento do Núcleo de Segurança do Paciente. Cogitare enferm (Online). 2016; 21(5):1-9. Doi: http://dx.doi.org/10.5380/ce.v21i5.45925

16. Velho JM, Treviso P. Implantação de programa de qualidade e acreditação: contribuições para a segurança do paciente e do trabalhador. Rev. Adm. Saúde. 2013;15(60):90-4.

17. Souza RFF, Silva LD. Estudo exploratório das iniciativas acerca da segurança do paciente em hospitais do Rio de Janeiro. Rev. enferm. UERJ (Online). 2014 [citado em 7 jan 2018]; 22(1):22-8. Doi: https://doi.org/10.12957/reuerj.2014.11399.

18.Schout D, Novaes HMD. Do registro ao indicador: gestão da produção da informação assistencial nos hospitais. Ciênc. saúde coletiva (Online). 2007 [citado em 12 dez 2017]; 12(4):935-44. Doi: http://dx.doi.org/10.1590/S1413-81232007000400015.

19.Souza LP, Bezerra AL, Silva AE, Carneiro FS, Paranaguá TT, Lemos LF. Eventos adversos: instrumento de avaliação do desempenho em centro cirúrgico de um hospital universitário. Rev. enferm. UERJ. 2011; 19(1):127-33.

20.Sousa MRG, Silva AEBC, Bezerra ALQ. Prevalência de eventos adversos em uma unidade de hemodiálise. Rev. enferm. UERJ (Online). 2016 [citado em 7 jan 2018]; 24(6):e18237. Doi: ttps:// doi.org/10.12957/reuerj.2016.18237.

21. The National Quality Forum. Safe Practices for Better Healthcare 2010 update. Washington (DC): The National Quality Forum; 2010.

22. Lima CSP, Barbosa SFF. Ocorrência de eventos adversos como indicadores de qualidade assistencial em unidade de terapia intensiva. Rev. enferm. UERJ (Online). 2015 [citado em 7 jan 2018]; 23(2):222-8. Doi: https://doi.org/10.12957/reuerj.2015.6076.

23. Aveling EL, Kayonga Y, Nega A, Dixon-woods M. Why is patient safety so hard in low-income countries? A qualitative study of healthcare workers' views in two African hospitals. Global Health[Online]. 2015; 11(6):1-8. Doi: 10.1186/s12992015-0096-x.

24. Reason J. Human error: models and management. BMJ. 2000; 320(7237):768-70.

25. Lorenzini E, Santi JAR, Báo ACP. Segurança do paciente: análise dos incidentes notificados em um hospital do sul do Brasil. Rev. gaúcha enferm. 2014; 35(2):121-7.

26. Fins JJ. What's wrong with evidence-based medicine? Hastings Center Report (Online). 2016 [cited in 2017 Apr 04]; 46(1):1. Doi: $10.1002 /$ hast.534.

27. Cohen T. Reevaluating recovery: perceived violations and preemptive interventions on emergency psychiatrics rounds. J. Am. Med. Inform. Assoc (Online). 2007; 14(3):312-9. Doi: 10.1197/jamia.M2245.

28. Kawamoto AM, Oliveira JLC, Tonini NS, Nicola AL. Liderança e cultura de segurança do paciente: percepções de profissionais em um hospital universitário. Rev. pesqui. cuid. fundam (Online). 2016 [citado em 7 jan 2018]; 8(2):4387-98. Doi: http://dx.doi. org/10.9789/2175-5361.2016.v8i2.4387-4398.

29. Reis WG, Scherer MDA, Carcereri DL. O trabalho do cirurgião-dentista na atenção primária à saúde: entre o prescrito e o real. Saúde debate (Online). 2015 [citado em 7 abril 2017]; 39(104):5664. Doi: http://dx.doi.org/10.1590/0103-110420151040608. 